\title{
Gerdy tubercle osteotomy in surgical approach of posterolateral corner of the knee
}

\author{
Raffaele Garofalo • Michael Wettstein • \\ Greg Fanelli $\cdot$ Elyazid Mouhsine
}

Received: 10 October 2005 / Accepted: 27 January 2006/ Published online: 2 September 2006

(C) Springer-Verlag 2006

\begin{abstract}
Posterolateral corner (PLC) injuries of the knee are uncommon injury patterns that may result in significant degrees of functional disability. When surgery is necessary to address this injury a good knowledge of anatomy and a good surgical exposure is the mainstay. This report reviews the complex anatomy of the posterolateral corner (PLC) of knee and describes the osteotomy of Gerdy tubercle as a technical variant to approach this anatomical region. This surgical step allowing a good mobilisation of the multiple layers of ilio-tibial tract from distal to proximal makes an excellent exposure of the PLC structures with absence of morbidity.
\end{abstract}

Keywords Gerdy tubercle - Posterolateral corner · Knee

\section{Introduction}

In recent years, the posterolateral corner (PLC) of knee has received considerable attention in terms of elucidation of its anatomy and assessment of its biomechanical role in the stabilisation of the knee joint.

\footnotetext{
R. Garofalo · M. Wettstein

Department of Orthopedics and Traumatology,

University Hospital of Lausanne, Lausanne, Switzerland

G. Fanelli

Department of Orthopaedic Surgery, Geisinger Medical Center, Danville, PA, USA

E. Mouhsine $(\square)$

OTR-BH 14, CHUV 1011 Lausanne, Switzerland

e-mail: Elyazid.mouhsine@chuv.ch
}

The PLC is composed of the iliotibial band, biceps, lateral collateral ligament (LCL), popliteus complex, fabellofibular ligament (FFL), popliteofibular ligament (PFL), arcuate ligament, coronary ligament, posterior horn of the lateral meniscus, middle third of the lateral capsular ligament, and posterolateral joint capsule [4, 16]. Seebacher et al. [21] postulated three distinct layers of the PLC. The most superficial layer consists of the iliotibial band and biceps femoris tendon. The peroneal nerve is located just deep to the biceps femoris tendon within this layer. The second, or middle layer consists of the quadriceps retinaculum and patellomeniscal ligament anteriorly and two patellofemoral ligaments posteriorly. The third, or deep layer is functionally most important and consists of a superficial and deep capsular lamina. The superficial lamina includes anteriorly the LCL and posteriorly the FFL. The deep lamina consists of coronary ligament, popliteus complex, PFL and arcuate ligament. Injuries involving PLC structures have been found to be one of the most debilitating ligament injuries of knee, and when missed may significantly affect the outcome of cruciate ligaments reconstruction. The diagnosis and management of PLC injuries remain difficult. Various surgical techniques have been developed to treat them $[1-3,6,7,10,12,13,15,22,25,26]$, however understanding of anatomy and surgical approach are very helpful to undertake reconstruction. The most popular surgical approach to PLC of knee is the one described by Terry and LaPrade [24] and consists of three fascial incisions and one lateral vertical capsular incision. The purpose of this paper is to review the complex anatomy of the PLC and describe a technical variant to approach it, consisting in osteotomy of Gerdy tubercle allowing a good mobilisation of the iliotibial tract, 
which in turn allows excellent exposure of the PLC structures.

\section{Surgical approach}

The patient is placed in the supine position on the operating room table. The injuried knee is flexed $45^{\circ}$ on a leg holder, and skin landmark is traced. A hockey stick incision extending from the midlateral aspect of the distal thigh over the lateral femoral epicondyle, along the iliotibial band, and distally past Gerdy tubercle, midway between it and anterior head of fibular head, is made. The anterior, posterior skin and subcutaneous tissue flaps are raised to allow for adequate exposure the iliotibial band and biceps femoris muscle complex. The Gerdy tubercle is identified, and with an electrocautery the lines of osteotomy are marked. A $10 \mathrm{~mm}$ wide osteotomy is oriented parallel to the long axis of the tibia and is advanced to detach a $10 \mathrm{~mm}$ distally based, $20 \mathrm{~mm}$ long and $7-10 \mathrm{~mm}$ thick bone flap from the Gerdy tubercle. Before complete bone release, the surgeon predrills the screw hole for later fixation with a 4 or $6.5 \mathrm{~mm}$ cancellous screw. Detachment of the tubercle of Gerdy and reflection upward of the iliotibial band allows access to the deep PLC layers. The iliotibial band has four different attachments at the knee $\% \%$, but essentially we recognized one superficial and one deep layer. The superficial layer is the most anterior and is first encountered after dissecting through the subcutaneous tissues on the lateral aspect of the knee. Kaplan [8] named it the iliotibial "band". It represents a pure iliotibial connection that attaches to Gerdy tubercle and extending freely past the femur to the hip where it terminates in a muscle belly. The iliopatellar "band" is the anterior expansion of the superficial layer that attaches to the lateral border of the patella. This band has an important role in patellofemoral tracking.

The posterior layer, named iliotibial "tract" [19], represents the deep portion of the iliotibial band [18]. It extends from the medial aspect of the superficial layer, is attached to the intermuscular septum of the distal femoral condyle proximal to the LCL, average $5 \mathrm{~cm}$ proximal to the lateral epicondyle, blends with fascial attachment of the long head of biceps, and contains femorotibial ligamentous fibers, representing the anterolateral femorotibial ligament (ALFTL) [19]. The ALFTL extends from the linea aspera of the femur to the Gerdy tubercle and acts as an anterolateral sling to the knee. A capsuloosseous layer of iliotibial tract deeper than these layers has been described $[17,20,24]$. It's a fascial layer, beginning proximally as the lateral investing fascia of the lateral gastrocnemius tendon and the medial investing fascia of the short head of biceps femoris muscle. This layer merged via a fascial extension, with the short head of biceps femoris, courses distally along the lateral femoral condyle and further merging with posterior parts of the superficial layers, posterior deep layer (iliotibial "tract"), inserting onto the lateral tibial tuberosity just posterior and proximal to Gerdy tubercle [24]. The knowledge of this complex anatomy of iliotibial band and relationship with the short head of biceps femoris muscle is very important to perform this surgical approach. In fact, to obtain a correct surgical exposure, the tubercle of Gerdy with the insertion of the iliotibial tract must be detached all the way to the synovial capsule at the border of the cartilage, so that the innermost deep fibers, which are the most effective in terms of femorotibial stabilisation, remain attached to the fragment. When the fragment at last stage of procedure is repositioned, normal lateral stability is restored. In particular it is very important to verify whether the connection of the iliotibial "tract" to the proximal femoral condyle remains intact toward the lateral lip of the linea aspera, otherwise it must be attached. Once the tubercle of Gerdy is detached and superficial and deep structures of iliotibial band dissected and reflected upward it is temporarly attached with two stitches at the upper border of the skin incision. Through this surgical approach we have a good exposure to capsular layer, the mid-third lateral capsular ligament (front), LCL, meniscofemoral portion of the posterior capsule (back), anterior arms and lateral aponeuroses of the long and short heads of the biceps femoris, capsular arm of the short head of biceps femoris and lateral gastrocnemius tendon posteriorly [11]. The LCL and popliteus tendon femoral attachment sites are easily identified with a gloved finger. A lateral transverse incision at the level of superficial capsular lamina, from front to the back, starting $1 \mathrm{~cm}$ anterior to an imaginary line parallel to the fibular shaft is done and so just back to femoral condyle, this window allows visualisation of the middle third of the capsular ligament, insertion of popliteus tendon, LCL, eventually the FFL extending the knee, and the deep lamina containing meniscofemoral capsule posteriorly, posterior part of the lateral meniscus, coronary ligament, popliteal complex and upper part of arcuate ligament . The arcuate ligament is a Y-shaped layer of connective tissue that originates from the tip of fibular styloid process just lateral, superior and superficial to the PFL, diverges into a medial and a lateral limb, and reinforces the posterolateral aspect of the capsule [14]. The incision of superficial lamina, particularly in the 
chronic case can be very thickened and of a bursal layer, often encountered in this region [2] allows to identify the LCL origin that is the lighthouse of PLC reconstruction. Two vertical capsular arthrotomies are performed. The first anterior arthrotomy is performed in a vertical fashion anterior to LCL, through the midgirth lateral capsule directly to over the femoral popliteal sulcus [23] to expose the distal condylar articular surface and peripheral edge of the anterolateral lateral meniscus. With a gentle retraction, the popliteus tendon can be visualised, when present in its oblique course. A second deep posterior incision for arthrotomy is performed vertically behind the LCL at the level of posterior meniscofemoral capsule in front of gastrocnemious tendon. Through this second incision posterior part of the lateral meniscus and popliteus meniscal fascicles, coronary ligament and upper part of arcuate ligament can be approached [11]. Sometimes, in case of acute lesions, it might not be not necessary to perform capsular incision because it is disrupted by the trauma so a direct access to deep structures is immediate after Gerdy tubercle osteotomy.

Noted that the LCL from its attachment site on the femur course on the lateral aspect of the knee deep to the iliotibial band and the reflected arm of the long head of the biceps femoris [18], the distal medial fibers of the LCL insert into the lateral edge of the fibular head merging with the tendon of the biceps femoris muscle, inserting lateral, inferior and anterior to the insertions of the arcuate ligament and the PFL [14]. The distal quarter of the LCL is exposed along both its lateral and anterior aspect within the fibular collateral ligament-biceps bursa [18] The lateral fibers of the LCL continue distally, medial to the anterior arm of long head of biceps tendon, blending with the superficial fascia of the lateral compartment of the leg. When necessary, overall in cases of PLC reconstruction, a second fascial incision (the third limb of the surgical triad described by Terry and LaPrade [24]) is then made posterior to the long head of biceps femoris and parallel to the peroneal nerve. After division of the superficial fascia, a fat stripe is often visible proximally, overlying the peroneal nerve. Meticolous dissection is carried out from proximal to distal so the nerve can be isolated, protected and eventually neurolysed to mobilise it. Through this interval is possible to access to the posteromedial aspect of the fibular styloid and posterolateral aspect of tibia. Retracting anteriorly the long head of biceps, and posteriorly the lateral head of gastrocnemius muscle, we can address the PFL attachment site, FFL, lower part of popliteus corner structures, medial and lateral limb of arcuate ligament. A further 3-4 cm length incision on the fibular head a little bit anterior to the central origin of LCL $[2,10]$ can be necessary to perform fibular tunnel in cases in which a reconstruction of LCL or PFL is carried out. Once PLC repair or reconstruction with whatever surgical technique is carried-out, the capsule is closed in a running fashion and Gerdy tubercle is fixed into your original bed. The fixation is performed with the knee in $20^{\circ}$ of flexion and slight valgus to maximally reduce tension on the iliotibial band, and a cancellous screw with a plastic washer is tightened.

\section{Discussion}

The anatomy of PLC of knee is very complex and to perform a surgical treatment of this kind of lesion a good knowledge and an optimal surgical approach is necessary. Different articles have contributed to elucidate the anatomic details of this region $[2,4,5,11,14$, $18,19]$ from which until today two detailed surgical approaches were developed and described [7, 24]. The most popular surgical approach to PLC of knee was described by Terry and LaPrade [24], who proposed a three fascial incisions and one lateral vertical capsular incision. The idea of Gerdy tubercle osteotomy to approach PLC of knee is not new [19], however it is not yet well described in literature. The rationale of this exposure is that the deep structures of PLC can be clearly addressed by retracting the iliotibial band complex together with its tubercle of insertion upward to its attachment on the femoral condyle. Sometimes, the superficial layer of iliotibial band can be injuried with a PLC injury, so during acute repair the surgical approach is carried-out through it [9]. When we address chronic injuries or the major part of acute lesion, this passage is lacking. With tubercle of Gerdy osteotomy, a careful dissection of deep layers of iliotibial band complex from deeper structures, allows directly a good exposure to anterior arms and lateral aponeuroses of long and short head of biceps, mid-third lateral capsular ligament, meniscofemoral portion of the posterior capsular layer, and also capsular arm of the short head of biceps. To obtain the same level of exposure through iliotibial tract splitting, a posterior retraction of superficial layer of iliotibial band is necessary to visualise deep and capsuloosseous layers, which should be dissected separately from the posterior aspect of the intermuscular septum and lateral gastrocnemius tendon respectively, to allow more posterior retraction of the posterior half of the tibial tract, so an evaluation of the meniscofemoral portions of the posterior capsule and capsular arm of the short head of biceps is possible [24]. Another practical 


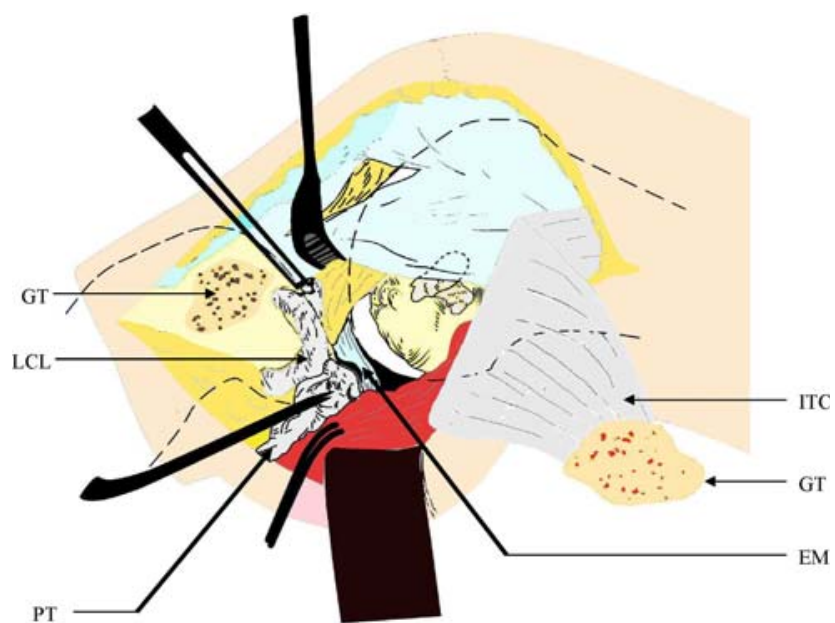

Fig. 1 Schematic drawing showing as the Gerdy tubercle $(G T)$ detachement associated with a proximal reflection of iliotibial compex (ITC) allows an early access to posterolateral corner structures: LCL (lateral collateral ligament), external meniscus $(E M)$ and popliteal tendon $(P T)$

advantage of this technical trick, is that when a PLC reconstruction is performed, whether with isometric or anatomic techniques, the grafts are passed from proximal fibula to the lateral femoral epicondyle beneath iliotibial tract and biceps femoris, so if the iliotibial tract retracts all these passages to make it easier and very visible.

In no manner this technique seems to alter the feeling of obtained PLC repair or reconstruction. In fact, the function of anterior superficial half of iliotibial

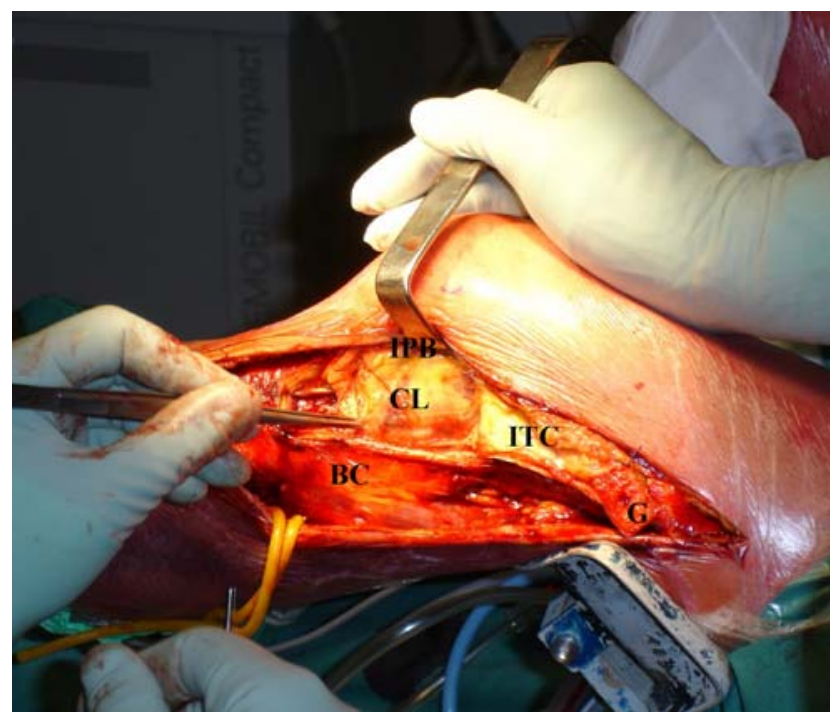

Fig. 2 Detachment of Gerdy tubercle $(G)$ and iliotibial complex $(I T C)$ associated with iliopatellar band (IPB) reflection, reveals the capsular layer $(C L)$ of the knee, and allows a good access to biceps complex $(B C)$

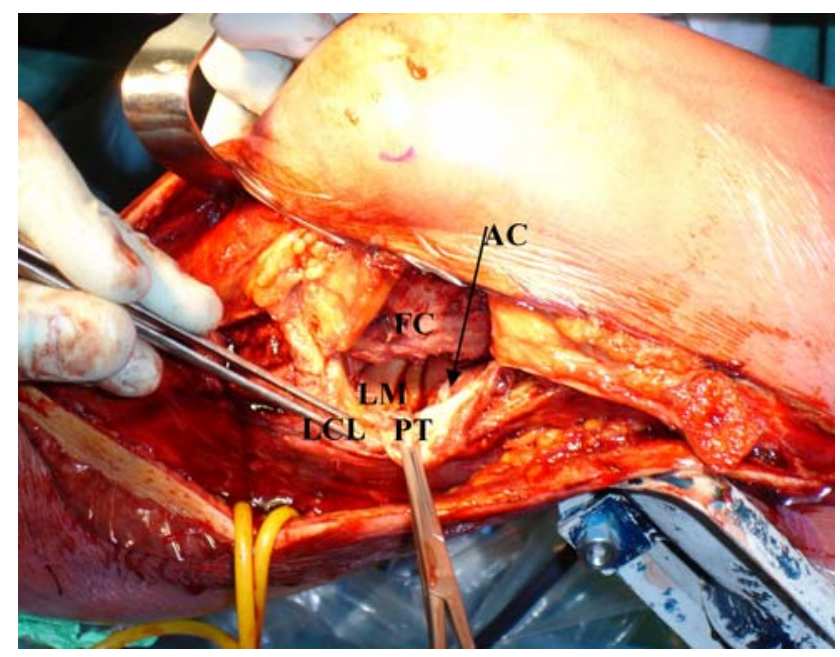

Fig. 3 Vertical incision of lateral capsule, in this acute injury revealed the ruptured popliteal tendon $(P T)$ and lateral collateral ligament $(L C L)$. Lateral meniscus $(L M)$ and femoral condyle $(F C)$ with site of LCL and PT attachment is also well exposed. In the posterior part of deep incision the upper part of arcuate ligament $(A C)$ can be revealed

band (iliotibial band of Kaplan [8]) is to actively stabilise the lateral side, against varus stress, extend the knee between $0^{\circ}$ and $30^{\circ}$ and rotate it externally at a greater angle of flexion. The posterior half, iliotibial "tract", deep posterior portions of the iliotibial tract, contain ALFTL extending from the linea aspera of the femur to the Gerdy tubercle that contribute to anterolateral knee stability. In definitive, the detachment of Gerdy tubercle determines a varus laxity and an anterolateral rotatory instability of the knee joint,

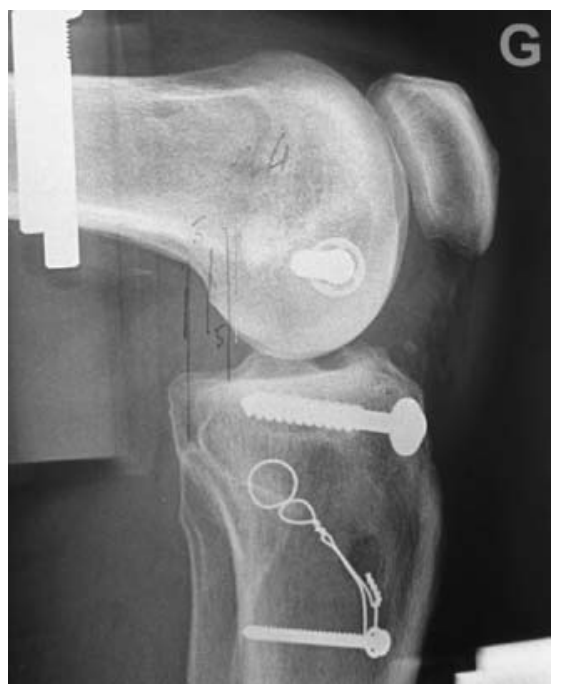

Fig. 4 Four months post-operative X-ray reveals a good union of Gerdy tubercle in these patients for whom ACL and PCL reconstruction were associated with a PLC repair 
without affecting the posterolateral stability so the reconstruction or repair of PLC can be tested without any bias at any time of procedure.

This surgical approach is relatively easy to perform and allows a very good exposure of structures of PLC of the knee. The absence of intra-operative and postoperative complications and the fact that this technical trick in no way has restricted post-operative rehabilitation and encourages the use of this procedure to approach the PLC of knee (Figs. 1, 2, 3, 4).

\section{References}

1. Albright JP, Brown AW (1998) Management of chronic posterolateral rotatory instability of the knee: Surgical technique for the posterolateral corner sling procedure. Instr Course Lect 47:369-378

2. Arciero RA (2005) Anatomic posterolateral corner knee reconstruction. Arthroscopy 21(9):1147.e1-1147.e5

3. Cooper DE (1999) Treatment of combined posterior cruciate ligament and posterolateral injuries of the knee. Oper Tech Sports Med 7:135-142

4. Covey DC (2001) Injuries of the posterolateral corner of the knee. J Bone Joint Surg 83A:106-118

5. De Maeseneer M, Shahabpour M, Vanderdood K, De Ridder F, Van Roy F, Osteoux M (2001) Posterolateral supporting structures of the knee: findings on anatomic dissection, anatomic slices and MR images. Eur Radiol 11(11):2170-7

6. Fanelli GC (2003) Evaluation and treatment of the multiple ligament injured knee. Arthroscopy Suppl 19:30-7

7. Hughston JC, Jacobsson KE (1985) Chronic posterolateral instability of the knee. J Bone Joint Surg 67A:351-359

8. Kaplan EB (1957) Surgical approach to the lateral (peroneal) side of the knee joint. Surg Gynecol Obstet 104:346356

9. LaPrade RF, Wentorf F (2002) Diagnosis and treatment of posterolateral knee injuries. Clin Orthop Relat Res 402:110 21

10. LaPrade RF, Johansen S, Wentorf FA, Engebretsen L, Esterberg JL, Tso A (2004) An analysis of an anatomical posterolateral knee reconstruction. Am J Sports Med 32:1405-1415

11. LaPrade RF, Konowalchuk BK (2005) Popliteomeniscal fascicle tears causing symptomatic lateral compartment knee. Am J Sports Med. 33(8):1231-6. Epub 2005 Jul 6
12. Latimer HA, Tibone JE, ElAttrache NS, McMahon PJ (1998) Reconstruction of the lateral of the lateral collateral ligament of the knee with patellar tendon allograft:Report of a new technique in combined ligament injuries. Am J Sports Med 26:656-662

13. Larson RV (2001) Isometry of the lateral collateral and popliteofibular ligaments and techniques for reconstruction using a free semitendinosus tendon graft. Oper Tech Sports Med 9: 84-90

14. Lee J, Papakonstantinou O, Brookenthal KR, Trudell D, Resnick DL (2003) Arcuate sign of posterolateral knee injuries: anatomic, radiographic, and MR imaging data related to patterns of injury. Skeletal Radiol 32:619-627

15. Martin SD, Clancy WG (1996) Posterolateral instability of the knee:treatment using the Clancy biceps femoris tenodesis. Oper Tech Sports Med 4:182-191

16. Maynard MJ, Deng X, Wickiewicz TL, Warren RF (1996) The popliteofibular ligament. Rediscovery of a key element in posterolateral stability. Am J Sports Med 24(3):311-6

17. McGuire DA, Wolchok JC (2003) Posterolateral corner reconstruction. Arthroscopy 19(7):790-3

18. Moorman CT III, LaPrade RF (2005) Anatomy and biomechanics of the posterolateral corner of theknee. J Knee Surg 18(2):137-45

19. Muller W (1983) In Muller W (ed) The knee: form, function and ligament reconstruction. Springer, Berlin Heidelberg New York, pp195-198

20. Noyes FR, Barber-Westin SD (1996) Surgical restoration to treat chronic deficiency of the posterolateral complex and cruciate ligaments of the knee joint. Am J Sports Med 24:415-426

21. Seenbacher JR, Inglis AE, Marshall JL, Warren RF (1982) The structure of the posterolateral aspect of the knee. J Bone Joint Surg 64A:536-41

22. Stannard JP, Brown SL, Farris RC, McGwin G Jr, Volgas DA (2005) The Posterolateral Corner of the Knee. Am J Sports Med 2005 Jun;33(6):881-8. Epub 2005 Apr 12

23. Stäubli HU, Birrer S (1990) The popliteus tendon and its fascicles at the popliteal hiatus:gross anatomy and functional arthroscopic evaluation with and without anterior cruciate ligament deficiency. Arthroscopy 6:209-220

24. Terry GC, LaPrade RF (1996) The posterolateral aspect of the knee. Anatomy and surgical approach Am J Sports Med 24:732-739

25. Veltri DM, Warren RF (1996) Treatment of acute and chronic injuries to the posterolateral and lateral knee. Oper Tech Sports Med 4:174-181

26. Verma NN, Mithofer K, Battaglia M, MacGillivray J (2005) The docking technique for posterolateral corner reconstruction. Arthroscopy 21(2):238-42 